\title{
Envisioning the future of the Journal of Anesthesiology \& Clinical Science
}

\author{
D. John Doyle \\ Correspondence: EIC-JACS@hoajonline.com \\ Department of General Anesthesiology, Cleveland Clinic, 9500 Euclid Avenue, E31, Cleveland, Ohio, 44195, USA.
}

\section{Editorial}

As we enter 2013 it seems appropriate to reflect on what the Journal of Anesthesiology \& Clinical Science has achieved over the last year. JACS has completed its inaugural volume (2012, Volume1) under an Open Access model that provides free and unrestricted access to all its published articles via Creative Commons license. Topics addressed by the journal include basic science and clinical aspects of anesthesiology, perioperative care, acute and chronic pain management, critical care, clinical and experimental research, the study of anesthetic molecular mechanisms, as well as patient monitoring, anesthesia technology and other issues.

Supported by an extensive editorial board, the journal has now published 15 articles and looks forward to greatly expanding this number in the future. As always, the journal's mission has been to provide freely accessible, top quality articles subjected to careful peer review. The rigor of our peer-review process is reflected in the journal's acceptance rate of about $30 \%$.

As noted above, JACS is a Creative Commons Open Access journal. Under this arrangement, the authors retain ownership of the copyright for their article, but authors allow anyone to download, reuse, re-print, modify, distribute, and/or copy articles, so long as the original authors and source are cited. (I have previously called this concept "Information Philanthropy" [1]). This means, for instance that a university instructor would be able to collate together a number of articles from JACS and other similarly licensed sources for free distribution for teaching medical students or residents, or for distribution at workshops and conferences.

As another example, consider the scenario where (for instance) the Education Committee of the Society for Airway Management (www.samhq.com) wants to make available a down loadable Airway Casebook for free distribution to their members. While the committee could write such a book de novo, it would be far easier to collate together a number of case reports published under an appropriate Creative Commons license, perhaps starting with Berg et al.'s fascinating case report of airway compression from a multinodular goiter [2]. One could also add a preface and table of contents, and make the derivative document available for free download, for example in PDF format, all in a matter of a mere few days.

Next, consider another scenario where an anesthesiologist would like to distribute a JACS article to his or her residents, but is concerned that the article has become a bit dated. One option would be to provide the original article, with the addition of a supplementary note with updated information. But because the version of the Creative Commons license used by JACS allows for modification of published articles, an alternative would be to independently update the article in question to produce a new derivative document, being careful, of course, to credit the original authors and source. These are some of the exciting possibilities offered by the Creative Commons Open Access model.

There are other exciting possibilities as well. A number of individuals argue that in addition to publishing the results of scientific investigations, scientists should also make their data available for post-publication scrutiny, for example in spreadsheet format [3]. Known as the Open Data movement, and operating in the same ethos as Open Source software and Open Access scholarly publications, its proponents want the raw data associated with scientific publications to be freely available, for example via Internet download. As a specific example, the authors of a study of acupuncture treatment for chronic headache [4] have made their raw data available online for those individuals who might wish to study it in detail[5].

Initiatives such as this raise a number of interesting issues. For instance, if a researcher reanalyzes posted experimental data from another individual and is able to produce some valuable new insight worth publishing, should the original researchers be given authorship in the resulting publication, or would a note of appreciation in the acknowledgement section suffice? Another issue concerns the use of such data by an investigator who suspects that the study may have involved a breach of ethics or utilized a protocol that would likely not be approved at the institution of the second investigator.

Of course, investigators may offer a number of reasons to avoid embracing such a notion. First, preparing and uploading the data in a standardized format involves extra effort and usually costs money. Second, some worry that other researchers may discover errors in the data or in its analysis. Third, some 
feel that they "worked hard" to collect the study data and may not see why the data should be made available to "just anyone". Finally, the researchers may have specific plans to examine the data using new or alternate methods at some future time, but if they make this data publicly available, other researchers may do exactly this, thus precluding the publication of further papers by the original team.

These concerns notwithstanding, the Open Data movement is slowing making progress $[6,7]$. For instance, the US National Institutes of Health $(\mathrm{NIH})$ now requires that scientists receiving grants of $\$ 500,000$ or more annually provide a "data-sharing plan" in the grant application [8].

Should JACS move in this direction, and if so how? I invite your comments. Write me at EIC-JACS@hoajonline.com

\section{Competing interest}

The author declares that he has no competing interests.

\section{Publication history}

Received: 12-Jan-2013 Published: 16-Jan-2013

\section{References}

1. Doyle DJ, Ruskin KJ and Engel TP: The Internet and medicine: past, present, and future. Yale J Biol Med 1996, 69:429-37. | PubMed Abstract | PubMed Full Text

2. Berg EV, Gomes HJ, Conturie CL and Wein RO: Case report of multinodular goiter and airway compression in a preeclamptic patient. Anesthesiology \& Clinical Science 2012, 1: 10. | Pdf

3. Vickers AJ: Whose data set is it anyway? Sharing raw data from randomized trials. Trials 2006, 7:15. | Article | PubMed Abstract | PubMed Full $\underline{\text { Text }}$

4. Vickers AJ, Rees RW, Zollman CE, McCarney R, Smith CM, Ellis N, Fisher $\mathrm{P}$ and Van Haselen R: Acupuncture for chronic headache in primary care: large, pragmatic, randomised trial. BMJ 2004, 328:744. | Article | PubMed Abstract | PubMed Full Text

5. Website

6. Tenopir C, Allard S, Douglass K, Aydinoglu AU, Wu L, Read E, Manoff M and Frame M: Data sharing by scientists: practices and perceptions. PLOS One 2011, 6:e21101. | Article | PubMed Abstract | PubMed Full Text

7. Dunn AG, Day RO, Mandl KD and Coiera E: Learning from hackers: opensource clinical trials. Sci Transl Med 2012, 4:132cm5. | Article | PubMed

8. National Institutes of Health. Frequently asked questions: data sharing. Accessed January 12, 2013 from Website

\section{Citation:}

Doyle D J: Envisioning the future of the Journal of Anesthesiology \& Clinical Science. journal of Anesthesiology and Clinical Science 2013, 2:3. http://dx.doi.org/10.7243/2049-9752-2-3 